\title{
Successful Surgical Treatment of an Infected Right Coronary Artery Aneurysm-to-Right Ventricle Fistula after Sirolimus-eluting Stent Implantation
}

\author{
Ken Kishida ${ }^{1,2}$, Hajime Nakaoka ${ }^{1}$, Satoru Sumitsuji ${ }^{1}$, Hideaki Nakatsuji ${ }^{1}$, Madoka Ihara ${ }^{1}$, \\ Yuhei Nojima ${ }^{1}$, Iichiro Shimomura ${ }^{2}$ and Yoshiyuki Nagai ${ }^{1}$
}

\begin{abstract}
The development of infected coronary aneurismal fistula following stenting seems exceedingly rare. A sirolimus-eluting stent (SES) was implanted in a 70-year-old male patient for acute coronary syndrome. His fever persisted despite treatment with adapted antibiotics. Coronary angiography and 16-multidetector row computed tomography demonstrated the huge right coronary aneurysm forming a fistula to the right ventricle. The aneurysm was excised by surgical treatment. Histopathological examination of the resected mass revealed leucocyte infiltration at the stent site, which lead to the diagnosis of mycotic aneurysm. SESs may play a potential role in locally blunting the innate response to bacterial agents.
\end{abstract}

Key words: sirolimus-eluting stent, infected aneurysm, fistula, local immunosuppression

(DOI: 10.2169/internalmedicine.46.6459)

\section{Introduction}

To date, coronary stenting for acute coronary syndrome has been widely used $(1,2)$. Complications following stent implantation include thrombosis, rupture, sepsis and infected coronary aneurysm $(1,2)$. Among them, infected coronary aneurysm causes fatal outcome. In addition to contamination at the time of stent delivery, there are other mechanisms how stents can become infected, including transient bacteremia from skin flora via access-site hematomas, pseudoaneurysms, delayed bleeding, prolonged arterial sheath insertion, and several procedures performed from the same access site over a short time period (1). However, prophylactic antibiotic administration does not appear to be conducted routinely prior to coronary angioplasty stenting, because the incident rate of stent infections was reported to be less than one in 10,000 cases (1-3). Recently, coronary artery aneurysm formation is considered as one of complications after the use of sirolimus-eluting stent (SES), which markedly reduces the restenosis rate after coronary angioplasty $(3,4)$. Development of mycotic aneurysms after stenting SES has only been reported in two cases $(5,6)$. Furtermore, the incidence of infected aneurismal fistula formation after stenting SES has not been presented. Here, we report a case of recurrent unknown fever who developed infected coronary aneurysm with fistula at the site of stent implantation, which was diagnosed by coronary angiography and 16multidetector row computed tomography (MDCT).

\section{Case Report}

A 70-year-old man was admitted to our hospital with sustained angina. His past medical histories were hypertension, diabetic mellitus, mild renal dysfunction (serum creatinine levels was $1.6 \mathrm{mg} / \mathrm{dL}$ ) and old cerebral infarction. Electrocardiogram (ECG) on admission revealed predominant ST segment changes in II, III, and aVF leads. Using the right trans-radial approach method, emergent coronary angiography demonstrated a 99\%-delayed tight stenosis in the proximal right coronary artery (RCA) (Fig. 1A), and significant stenosis in the proximal left anterior descending artery (LAD) (Fig. 2A) and the middle circumflex artery (LCX) (Fig. 2B). The patient was diagnosed with the culprit lesions

\footnotetext{
${ }^{1}$ Department of Cardiology, Rinku General Medical Center, Izumisano, Suita and ${ }^{2}$ Department of Metabolic Medicine, Graduate School of Medicine, Osaka University, Suita

Received for publication December 25, 2006; Accepted for publication February 26, 2007
}

Correspondence to Dr. Ken Kishida, kkishida@imed2.med.osaka-u.ac.jp 
A

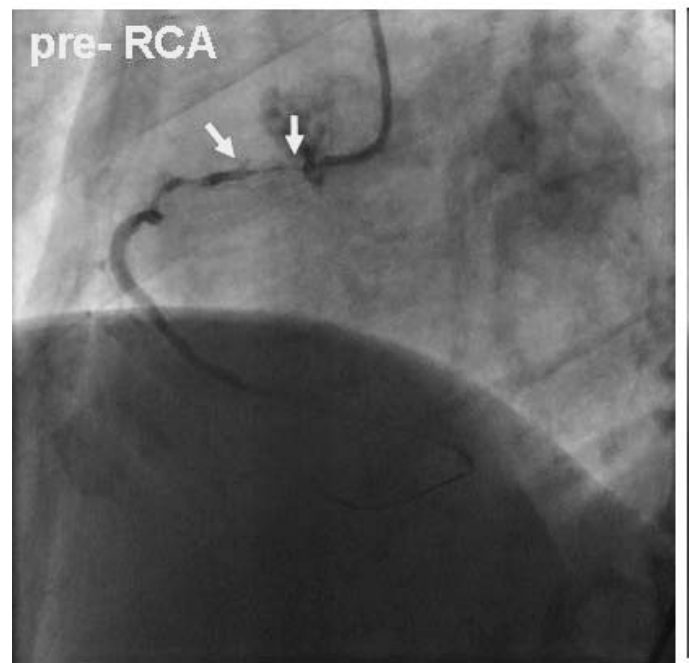

B

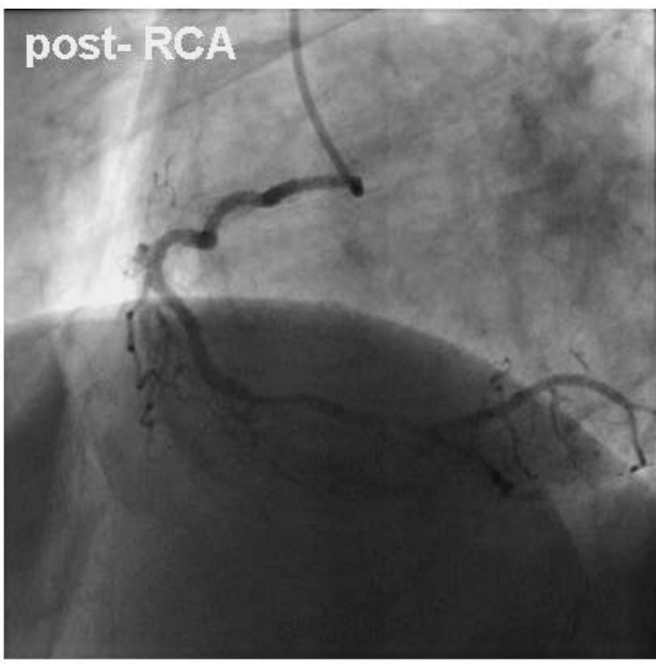

Figure 1. Right coronary artery angiogram performed during the initial procedure. Preangioplasty (left anterior oblique (LAO) view): the right coronary artery (RCA) was affected with severe stenosis in the proximal site (arrow) (A). The RCA was opened and a sirolimus-eluting stent (SES) was inserted (B). Satisfactory angiographic results were obtained with a smooth luminal outline. No contrast extravasation outside the vessel wall was noted on the image.
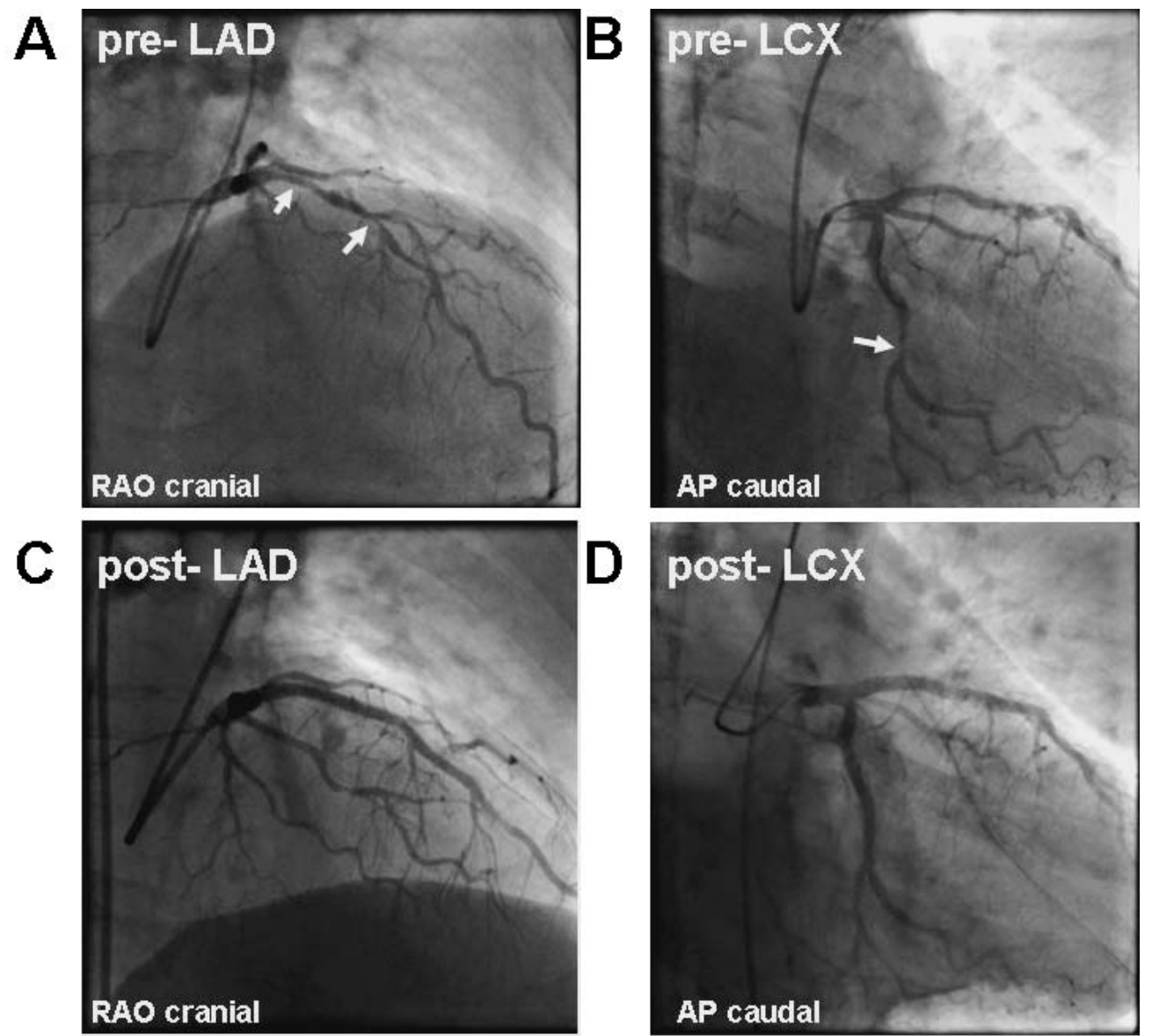

Figure 2. Left coronary artery (LCA) angiograms performed during the initial procedure. Preangioplasty (cranial and caudal views): significant stenosis (arrow) was also exhibited in the left anterior descending artery (LAD) (A) and the middle circumflex artery (LCX) (B). Both LAD and LCX were opened and each limus-eluting stent (SES) was inserted with a good immediate angiographic result $(C, D)$ after the appearance of lethal arrhythmias. 
of proximal RCA. It was difficult to hook RCA with various guide catheters because of a tortuous innominate artery. The lesion was barely crossed with an internal mammary artery catheter and universal guidewire by using the balloon anchor technique (7). The procedure, however, was cumbersome and prolonged because of vessel curvature and poor guiding catheter back up. Two sirolimus-eluting stents (SES, Cypher $^{\circledR}$ Cordis Johnson \& Johnson, Japan) $(2.5 \times 28$ and $2.5 \times 23 \mathrm{~mm}$ ) were deployed in the stenosed vessel (Fig. 1B) at 16 atm for 30 seconds. Incomplete dilatated RCA at the site of stent was postdilated with a $2.5 \mathrm{~mm}$ balloon. The volume of contrast used in this procedure was $189 \mathrm{~mL}$. Although the catheter of intravascular ultrasound (IVUS) did not finally cross the RCA lesions because of severe calcification of RCA tortuously, the end result was good without use of oversized balloon size, high-pressure inflation of the balloon, coronary dissection and contrast extravasation outside the vessel wall. Satisfactory angiographic results were obtained with a smooth luminal outline, and then the arterial sheath was removed.

The day after the procedure, the patient had fever, chills, malaise and no suppuration on the access site. Blood cultures yielded repeatedly Staphylococcus aureus ( $S$. aureus) and echography revealed neither valvular nor myocardial abnormalities. Urinalysis was negative and no line sepsis was found. Despite exhaustive screening for a potential source of the infection (including whole computed tomographic scan, transesophageal echocardiography, gastric endoscopic examination and gallium scintigraphy), no infectious focus was detected. The interventions for the residual left coronary artery (LCAs) lesions were necessary to perform. Intravenous adapted antibiotics was administrated, however, the patient's condition remained poor. A watchful waiting strategy, with aggressive intravenous antibiotic treatment, was selected because of progressive inflammatory signs and relapse of renal dysfunction (serum creatinine levels was $2.0 \mathrm{mg} / \mathrm{dL}$ ). Three weeks later he started to have fever, convulsion and syncope attack, accompanied by the torsades de pointes on ECG monitor. The causes were considered to be due to trouble of LCAs. Percutaneous transluminal coronary angioplasty of the LCAs was attempted. Two SESs $(3.0 \times 28$ and $3.0 \times 28$ $\mathrm{mm}$ ) were successfully implanted to completely cover the target LAD stenosis at 16 atm and post-dilate with $3.5 \mathrm{~mm}$ balloon (Fig. 2C). A SES $(3.0 \times 33 \mathrm{~mm})$ was inserted to the target LCX stenosis at 16 atm (Fig. 2D). The volume of contrast used was a minimum of $15 \mathrm{~mL}$ with IVUS. The regions were suspected to have occlusion or severe stenosis of RCA because final angiography demonstrated delayed retrograde filling images by collaterals from LCA to distal part of RCA. The patient had fever and renal dysfunction. Myocardium controlled by RCA was infracted and it was a concern that the increasing use of contrast volume would aggravate renal dysfunction due to the difficulty of percutaneous transluminal coronary angioplasty for tortuous RCA. The patient was subjected to coronary angioplasty to the LCAs alone. The postprocedural course was uneventful, and the in- flammatory sings were improved by the treatment with adapted antibiotics. The patient was transferred to a different hospital for the rehabilitation.

Two months later, the recurrence of high fever with eosinophilia led to a readimission to our hospital with the relapse of renal dysfunction (serum creatinine level was 3.7 $\mathrm{mg} / \mathrm{dL}$ ) and there was ST-segment depression in I, aVL, II, III and aVF leads on ECG. Clinical examination did not reveal any findings which suggested the cause of fever. There were no vegetations. The patient underwent a coronary angiogram as he had developed ischemia. The LCAs were unchanged compared with the previous final results (Fig. 3A), while the RCA was completely occluded at the stent portion (Fig. 3B-a), near the aneurismal dilatation (Fig. 3B-b) with the evidence of contrast spilling into the right ventricular chamber with rapid clearance (Fig. 3B-c). The volume of contrast used was $23 \mathrm{~mL}$. A Swan-Ganz catheter was subsequently inserted and right heart catheterization was performed for the oxygen saturation run. No significant step-up in oxygen saturation data was observed between the venous samples obtained from the vena cava and right ventricular, thereby concluding that the shunt fraction between the pulmonary and systemic (Qp/Qs) circulation was hemodynamically insignificant and less than 1.11 ratio at that particular time point. The pressure of the pulmonary artery, pulmonary artery wedge, right atrium, inferior vena cava and superior vena cava indicated 46/16 (30), 17, 49/6 (13), 10, 9 and $12 \mathrm{mmHg}$, respectively. The 16multidetector row computed tomography (MDCT) scan demonstrated the occlusion of proximal RCA (Fig. 4A-a, Fig. $4 \mathrm{~B}-\mathrm{a}$ and $-\mathrm{b})$, and a large aneurysm $(50 \times 30 \mathrm{~mm}$ in size, Fig. 4A-b, Fig. 4B-a and 4C) forming a fistula $(10 \mathrm{~mm}$ in size) between the aneurysm and right ventricle (Fig. 4A-c). The volume of contrast used on MDCT was $85 \mathrm{~mL}$. This coronary aneurismal fistulation was suggestive of an infectious focus. No revasularization was performed with coronary intervention. The decision was made to continue medical management because of the patient's complicated conditions. The fever persisted despite intravenous adapted antibiotic treatment. Finally, an elective operation was conducted. The surgical treatment consisted of stent removal and excision of the right coronary artery aneurysm without coronary artery bypass grafting. The right ventricular wall was reconstructed (Fig. 5A, 5B). Histopath-ological examination of the resected aneurismal wall revealed an extensive inflammatory reaction in the inner layer of the arterial wall, with destruction of the media and the formation of some microabscess [Fig. 5C (original magnification $\times 20$ ), 5D (original magnification $\times 200$ )]. Bacterial cultures of the resected stent showed no growth. The patient started to respond favorably to the antibiotics (Fig. 6). Infection parameters were normalized and the patient could be transferred to a different hospital for rehabilitation. There has been no recurrence of fever or angina in the past 16 months. 
A

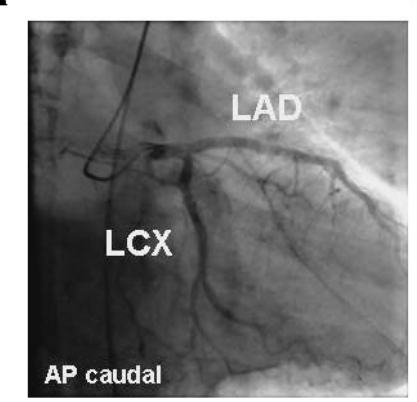

B
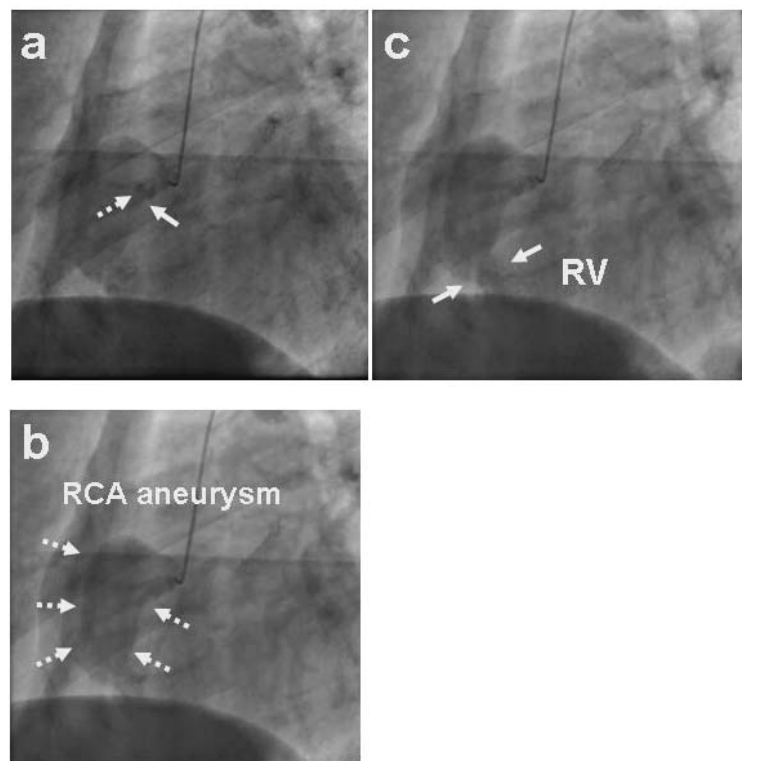

Figure 3. A, A coronary angiogram showed no change of left coronary arteries (LCAs) compared with the previous procedure. B, Angiography of the right coronary artery (RCA) demonstrated occlusion of proximal stent site (solid arrow), an entry point to aneurysm (dotted arrow) (a), and the saccular aneurysm (dotted arrow) in the area of proximal stents and its coronary artery fistula originating from the RCA stents (b), with contrast spillage into the right ventricular chamber (c) through the fistula (solid arrow). Rapid clearance of contrast was observed, with none in the pericardial space.

\section{Discussion}

The advancement of coronary intervention for coronary artery disease has provided cardiologists with a wider range of treatment (8). Development of intracoronary stents has facilitated a much less invasive therapy than open-heart surgery (9). Infection specificity, related to the use of intracoronary stents, is exceedingly rare. The reported infected coronary aneurysms at the stent sites are summarized in Table 1 $(5,6,10-16))$. Bacterial cultures of blood in the present case repeatedly grew $S$. aureus (Fig. 6). Of the 11 reports in the literature, including the present case, the potentially responsible bacteremia was either $S$. aureus $(\mathrm{n}=8)$ or Pseudomonas aeruginosa $(\mathrm{n}=2)$ (Table 1). The mechanism of sepsis remains unclear, because there had been no evidence of local access site infection following intervention. Mortality was fairly high, with death occurring in four of the eleven patients (Table 1). Myles et al reported a case managed solely with medical therapy (3). Five of seven patients treated surgically lived (Table 1). Therefore, if an infected coronary aneurysm is detected, prompt surgical excision of the stent and debridement of infected tissues is recommended to minimize the risk of rupture, cardiac tamponade and sudden death (10-13). The present case was quite rare in that the mycotic stented aneurysm formed a fistula to the cardiac chamber. This case is the first report of successful surgical treatment of an infected aneurismal fistula after SES implantation.
Why did infection develop in the present case at the site of sirolimus-eluting stenting? Sirolimus, belonging to rapamycin, has been used for systemic therapy in renal transplant patients (17). Sirolimus is capable of inhibiting not only elastic recoil and arterial remodeling caused by vascular injury, but also neointimal hyperplasia after angioplasty (9). The drug-eluting stent era was ushered in with the first published human study by Sousa et al in 2001, showing nearly complete abolition of neointimal hyperplasia by use of SES (18). In Japan, the use of SESs has been approved since 2004. Despite the dramatic capacity of SES to reduce the restenosis rate, SES-related local problems have been reported (9). First, the requirement of a prolonged dual antiplatelet regimen to avoid the risk of SES thrombosis is widely accepted (19). Second, the occurrence of acquired late malapposition in some patients suggests a continuous interaction of SES with the vessel wall promoting vessel remodeling. It has been recently reported that the implantation of a SES may cause late formation of coronary artery aneurysm (4). SES having an antiproliferative action may be responsible for the delayed and inappropriate healing, which likely leads to weakening of the arterial wall and delayed aneurysm formation (20). Third, local hypersensitivity has been documented in some patients suffering complications after SES implantation $(21,22)$. Virmani et al have shown that aneurismal dilatation of stented arterial segments is due to a severe localized hypersensitivity consisting predominantly of $\mathrm{T}$ lymphocytes and eosinophils (21), which is caused by the metallic stent, polymer, or sirolimus (21-23). 
A

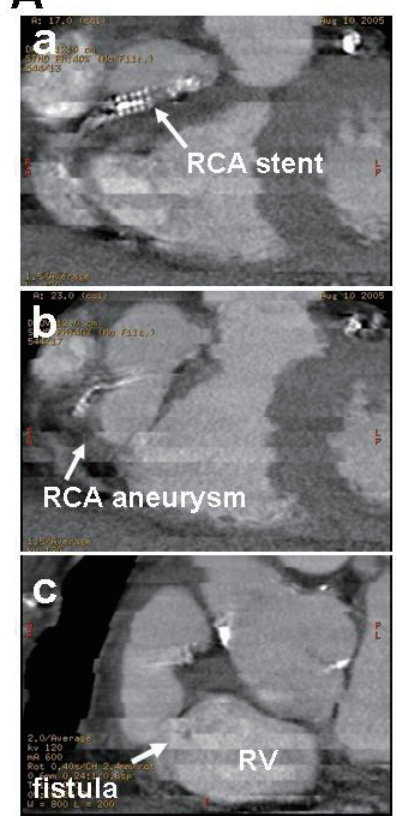

B

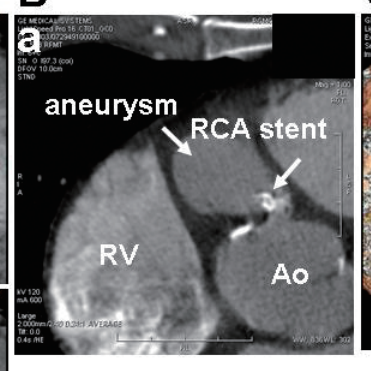

C

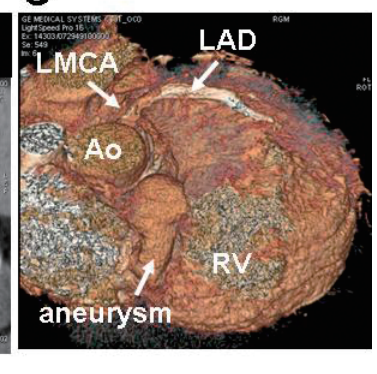

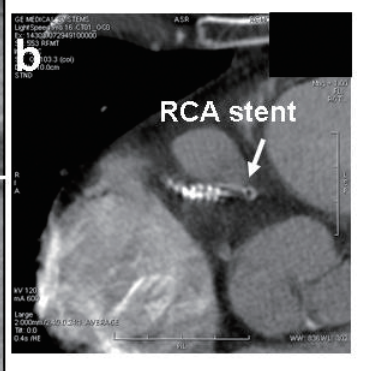

Figure 4. The 16-row multislice computed tomographic scan disclosed huge right coronary artery (RCA) aneurysm and draining image into right ventricular chamber via fistula. The large aneurysm was located between the proximal and distal areas of the stented region. The aneurysm did not connect to the stented lumen distal to the occlusion. The maximum intensity projection in longitudinal sections (A-a, b, c) and cross sections (B-a, b), and volume rendering (C) images of the markedly dilated RCA is indicated. The fistula foramen measured $10 \times 10 \mathrm{~mm}$ in diameter (A-c). Ao; ascending aorta, RV; right ventricle, LMCA; left main coronary artery, LAD; left anterior ascending artery.
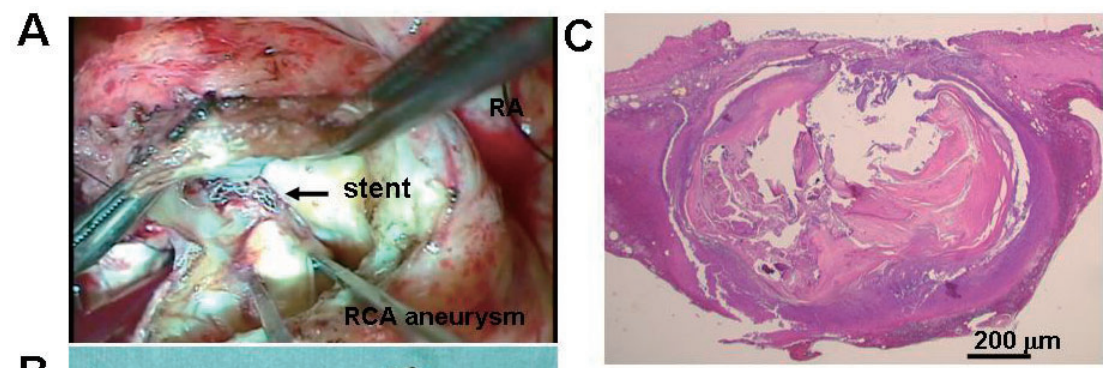

B

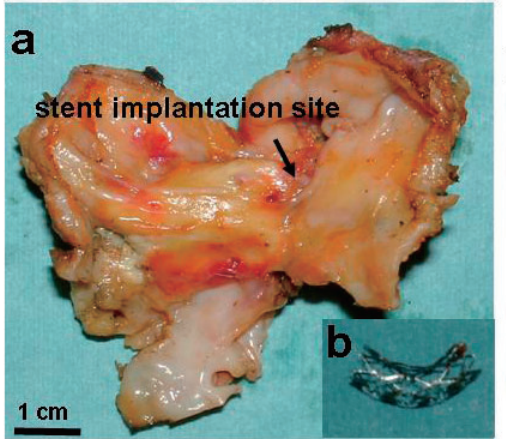

D

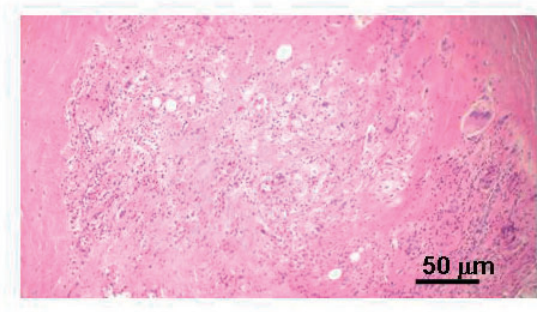

Figure 5. Intraoperative image of the coronary aneurysm before (A), and after excision and excised stent (B-b) and infected coronary aneurysm (B-a). Pathology of the false aneurysm after surgical excision (C). The aneurysm without neck measured $50 \times 30 \mathrm{~mm}$ in size and had a red appearance like normal vascular color. Its histopathologic examination demonstrated marked inflammatory reaction consisting of leucocytes within media (hematoxylin and eosin stain; original magnification $\times 20, \times 200$ ) without eosinophilic reaction.

We doubt that in the present case it was due to a similar mechanism, because the use of SES in LCAs showed no aneurismal formation (Fig. 3A) and there was no infiltration of eosinophilia in focus (Fig. 5C, 5D). Finally, could the ef- 


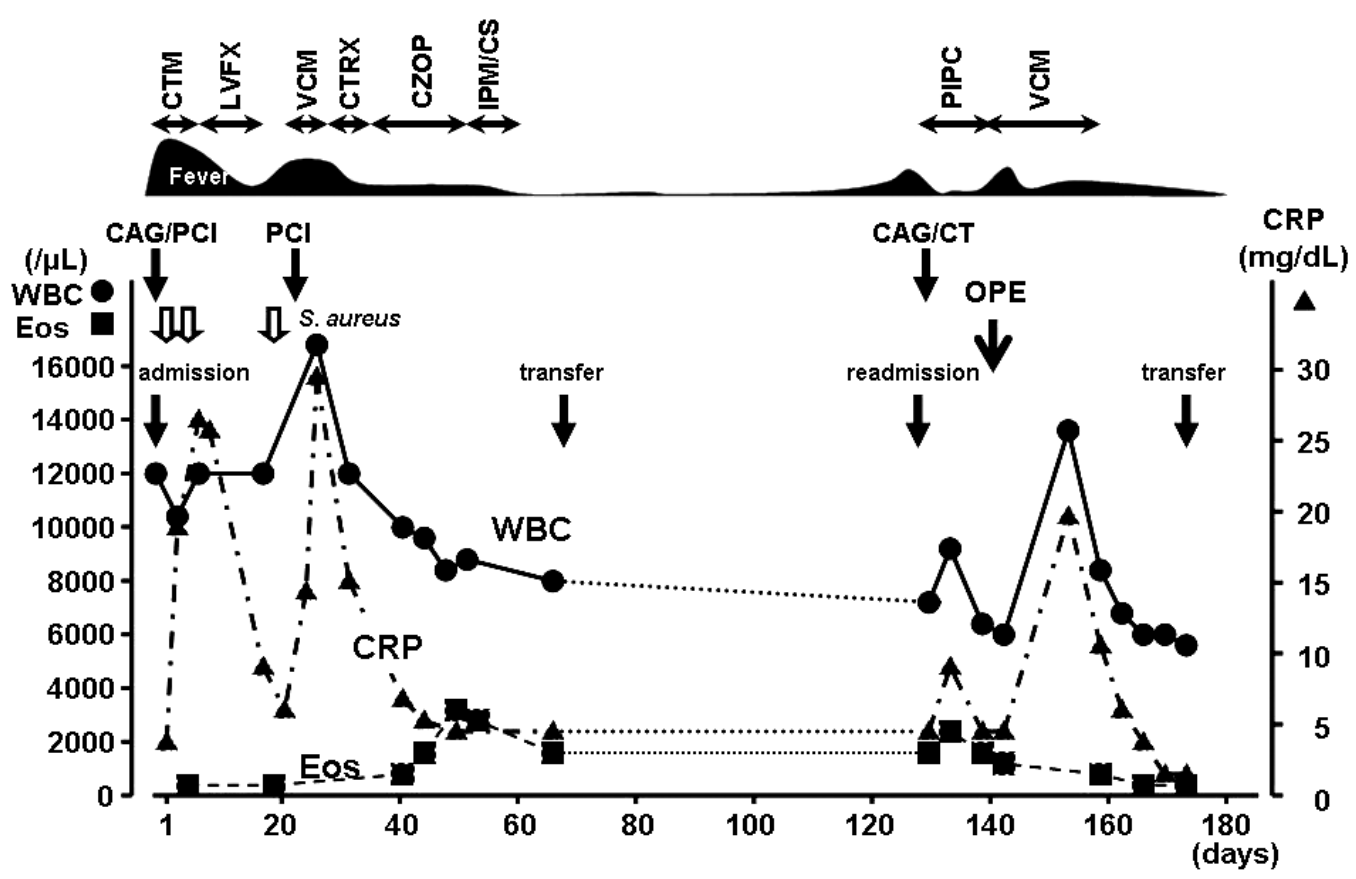

Figure 6. Clinical course, and white blood cell counts (WBC, filled circle), eosinophil counts (Eos, filled square), and C-reactive protein (CRP, filled triangle) levels in blood. OPE: operation, S. aureus; Staphlococcus aureus (open arrow). CAG: coronary angiogram, PCI: percutaneous coronary intervention. Each antibiotic, CTM; cefotiam, LVFX; levofloxacin, VCM; vancomycin, CTRX; ceftriaxone, CZOP; cefozopran, IMP/CS; imipenem/ cilastatin, PIPC; piperacillin.

Table 1. Infected Stented Coronary Aneurysm

\begin{tabular}{|c|c|c|c|c|c|c|c|c|c|}
\hline Case & Vessel & Stent & Fistula & Organism & Days & $\begin{array}{l}\text { Max } \\
\text { size }\end{array}$ & Ope & Outcome & Reference \\
\hline 66yo F & RCA & PS & - & S. aureus & 30 & 32 & + & Dead & 1993 Gunther \\
\hline 49yo M & LAD & PS & - & P. aeruginosa & 60 & - & + & Dead & 1996 Leroy \\
\hline 65уо M & RCA & PS & RA & unknown & 90 & - & + & Alive & 1996 Karim \\
\hline 38yo M & LCX & PS & - & $P$. aeruginosa & 10 & 16 & + & Alive & 1997 Bouchart \\
\hline 54yo M & LAD & MS & - & S. aureus & 3 & 17 & - & Dead & 1999 Grewe \\
\hline $67 y o ~ M$ & LCX & PS & - & S. aureus & 120 & - & - & Alive & 2000 Rensing \\
\hline 55yo M & LAD & $\mathbf{P}$ & - & S. aureus & 90 & 15 & + & Alive & 2005 Marcu \\
\hline 47yo M & RCA & $\mathbf{s}$ & - & S. aureus & 2 & - & - & Dead & 2005 Alfonso \\
\hline 56 yo M & LAD & $\mathbf{s}$ & - & S. aureus & 30 & 28 & + & Alive & ${ }_{2005}$ Singh \\
\hline 70yo M & RCA & $\mathbf{S}$ & RV & S. aureus & 120 & 50 & + & Alive & 2005 our case \\
\hline
\end{tabular}

PS: Palmaz-Schatz stent, MS: micro stent, P: paclitaxel eluting stent, S: sirolimus eluting stent. S.:Staphylococcus., P.: Pseudomonas., F: female, M: male, RCA: right coronary artery, LAD: left anterior descending artery, LCX: left circumflex artery, RA: right atrium, RV: right ventricle, Days:onset of aneurysm, Max size: max diameter of aneurysm, Ope: surgical operation

fects of sirolimus on the vessel wall at the site of SES implantation contribute to the development of infection? If infection does occur locally in the procedural period, it is likely that sirolimus might contribute to the development of aneurysms by suppression of the healing process and local immune response after artery injury. The cases of Alfonso et al (5) and Singh et al (6) developed the mycotic aneurysms at the site of SES implantation. Sirolimus has well-known effects on suppression of the immune response. The potential role of SES in locally blunting the innate response to bacterial agents may be considered. The formation of mycotic aneurismal fistula in the present case may be partly accounted for by this mechanism.

In conclusion, the objective signs of infection (unexplained fever, late or relapsing bacteremia) following coronary stenting should motivate cardiologists to perform noninvasive diagnosis, MDCT, to determine coronary infective aneurysm either with or without fistula.

We thank Osamu Monta, MD PhD and Yoshikado Sasako, MD 
$\mathrm{PhD}$ (Department of Cardiovascularsurgery, Rinku General Medical Center, Izumisano) for the excellent surgical treatment. We are also grateful to Syuuji Nakahira and Kumiko Nagahama from our hospital (Rinku General Medical Center, Izumisano) for their image processing technological assistance.

\section{References}

1. Baddour LM, Bettmann MA, Bolger AF, et al. Nonvalvular cardiovascular device-related infections. Clin Infect Dis 38: 11281130, 2004

2. Berkalp B, Kervancioglu C, Oral D. Coronary artery aneurysm formation after balloon angioplasty and stent implantation. Int J Cardiol 69: 65-70, 1999.

3. Myles O, Thomas WJ, Daniels JT, Aronson N. Infected endovascular stents managed with medical therapy alone. Catheter Cardiovasc Interv 51: 471-476, 2000.

4. Abreu L, Meireles GC, Forte AA, Sumita M, Hayashi J, Solano J. Coronary artery aneurysm one year and five months after sirolimus-eluting stent placement. Arq Bras Cardiol 85: 340-342, 2005 (in Portuguese).

5. Alfonso F, Moreno R, Vergas J. Mycotic aneurysms after sirolimus-eluting coronary stenting. Catheter Cardiovasc Interv 67: 327-328, 2006

6. Singh H, Singh C, Aggarwal N, Dugal JS, Kumar A, Luthra M. Mycotic aneurysm of left anterior descending artery after sirolimus-eluting stent implantation: a case report. Catheter Cardiovasc Interv 65: 282-285, 2005.

7. Fujita S, Tamai H, Kyo E, et al. New technique for superior guiding catheter support during advancement of a balloon in coronary angioplasty: the anchor technique. Catheter Cardiovasc Interv 59: 482-488, 2003.

8. Sigwart U, Puel J, Mirkovitch V, Joffre F, Kappenberger L. Intravascular stents to prevent occlusion and restenosis after transluminal angioplasty. N Engl J Med 316: 701-706, 1987.

9. Ong AT, Serruys PW. Drug-eluting stents: current issues. Tex Heart Inst J 32: 372-377, 2005.

10. Gunther HU, Strupp G, Volmar J, von Korn H, Bonzel T, Stegmann T. Coronary stent implantation: infection and abscess with fatal outcome. Z Kardiol 82: 521-525, 1993 (in German).

11. Leroy O, Martin E, Prat A, et al. Fatal infection of coronary stent implantation. Cathet Cardiovasc Diagn 39: 168-170, 1996.

12. Karim MA. Coronary artery aneurysmal fistula: a late complication of stent deployment. Int J Cardiol 57: 207-209, 1996.

13. Bouchart F, Dubar A, Bessou JP, et al. Pseudomonas aeruginosa coronary stent infection. Ann Thorac Surg 64: 1810-1813, 1997.

14. Grewe PH, Machraoui A, Deneke T, Muller KM. Suppurative pancarditis: a lethal complication of coronary stent implantation. Heart 81: 559, 1999.

15. Rensing BJ, van Geuns RJ, Janssen M, Oudkerk M, de Feyter PJ. Stentocarditis. Circulation 101: 188-190, 2000.

16. Marcu CB, Balf DV, Donohue TJ. Post-infectious pseudoaneurysm after coronary angioplasty using drug eluting stents. Heart Lung Circ 14: 85-86, 2005.

17. Groth CG, Backman L, Morales JM, et al. Sirolimus (rapamycin)based therapy in human renal transplantation: similar efficacy and different toxicity compared with cyclosporine. Sirolimus European Renal Transplant Study Group. Transplantation 67: 1036-1042, 1999.

18. Sousa JE, Costa MA, Abizaid AC, et al. Lack of neointimal proliferation after implantation of sirolimus-coated stents in human coronary arteries: a quantitative coronary angiography and threedimensional intravascular ultrasound study. Circulation 103: 192195, 2001.

19. McFadden EP, Stabile E, Regar E, et al. Late thrombosis in drugeluting coronary stents after discontinuation of antiplatelet therapy. Lancet 364: 1519-1521, 2004.

20. Degertekin M, Serruys PW, Tanabe K, et al. Long-term follow-up of incomplete stent apposition in patients who received sirolimuseluting stent for de novo coronary lesions: an intravascular ultrasound analysis. Circulation 108: 2747-2750, 2003.

21. Virmani R, Guagliumi G, Farb A, et al. Localized hypersensitivity and late coronary thrombosis secondary to a sirolimus-eluting stent: should we be cautious? Circulation 109: 701-705, 2004.

22. Nebeker JR, Virmani R, Bennett CL, et al. Hypersensitivity cases associated with drug-eluting coronary stents: a review of available cases from the Research on Adverse Drug Events and Reports (RADAR) project. J Am Coll Cardiol 47: 175-181, 2006.

23. Stabile E, Escolar E, Weigold G, et al. Marked malapposition and aneurysm formation after sirolimus-eluting coronary stent implantation. Circulation 110: 47-48, 2004.

(C) 2007 The Japanese Society of Internal Medicine http://www.naika.or.jp/imindex.html 